\title{
Molecular diffusion in monolayer and submonolayer nitrogen
}

\author{
Hansen, Flemming Yssing; Bruch, Ludwig Walter
}

Published in:

Physical Review B Condensed Matter

Link to article, DOI:

10.1103/PhysRevB.64.045413

Publication date:

2001

Document Version

Publisher's PDF, also known as Version of record

Link back to DTU Orbit

Citation (APA):

Hansen, F. Y., \& Bruch, L. W. (2001). Molecular diffusion in monolayer and submonolayer nitrogen. Physical Review B Condensed Matter, 64(4), 045413. https://doi.org/10.1103/PhysRevB.64.045413

\section{General rights}

Copyright and moral rights for the publications made accessible in the public portal are retained by the authors and/or other copyright owners and it is a condition of accessing publications that users recognise and abide by the legal requirements associated with these rights.

- Users may download and print one copy of any publication from the public portal for the purpose of private study or research.

- You may not further distribute the material or use it for any profit-making activity or commercial gain

- You may freely distribute the URL identifying the publication in the public portal

If you believe that this document breaches copyright please contact us providing details, and we will remove access to the work immediately and investigate your claim 


\title{
Molecular diffusion in monolayer and submonolayer nitrogen
}

\author{
F. Y. Hansen ${ }^{1}$ and L. W. Bruch ${ }^{2}$ \\ ${ }^{1}$ Department of Chemistry, Technical University of Denmark, IK·207·DTU, DK-2800, Lyngby, Denmark \\ ${ }^{2}$ Department of Physics, University of Wisconsin-Madison, Madison, Wisconsin 53706 \\ (Received 13 November 2000; revised manuscript received 28 March 2001; published 5 July 2001)
}

\begin{abstract}
The orientational and translational motions in a monolayer fluid of physisorbed molecular nitrogen are treated using molecular dynamics simulations. Dynamical response functions and several approximations to the coefficient of translational diffusion are determined for adsorption on the basal plane surface of graphite and on a fictitious uncorrugated graphite surface. The rotational diffusion constants in the plastic crystal and in the fluid phase are calculated. The possibility of observing these phenomena with quasielastic scattering experiments is discussed. The wave vector range is determined where the ballistic approximation to the translational molecular self-correlation function is accurate.
\end{abstract}

DOI: 10.1103/PhysRevB.64.045413

PACS number(s): 68.43.Pq, 68.35.Rh, 63.22.+m

\section{INTRODUCTION}

Although most of the data for monolayers of molecular nitrogen ${ }^{1-5}$ are for solids, the recent extensions of quasielastic neutron ${ }^{6-8}$ and helium atom ${ }^{9}$ scattering experiments raise the prospect that data on the dynamics of the fluid phases may become available. Here, we characterize the diffusive motions in monolayer $\mathrm{N}_{2}$ fluids using molecular dynamics simulations and explore how these motions are reflected in the correlation functions for inelastic scattering. We take advantage of the detail with which fluids of this relatively simple diatomic molecule may be modeled, but the methods of analysis apply also to disordered monolayer phases of more complex molecules. The dynamics of monolayer motions are complicated enough that simulation results are likely to be useful in the analysis of data for monolayer molecular fluids.

Rotational diffusion in the plastic two-dimensional (2D) $\mathrm{N}_{2}$ crystal is also treated. Although it has not been measured for monolayer $\mathrm{N}_{2}$, related experiments were performed for monolayer $\mathrm{CH}_{4} \cdot{ }^{10}$ The analysis of rotational diffusion here has application to the modeling of related phenomena arising in experiments on more complex fluids. ${ }^{8}$

Quasielastic neutron scattering (QNS) from adsorbed films has thus far been performed on hydrogenous materials, exploiting the large incoherent cross section of hydrogen. In contrast, low-energy neutron scattering from ${ }^{14} \mathrm{~N}$ is dominated by the coherent cross section. Quasielastic helium atom scattering (QHAS) has concentrated on low-coverage fluids where the self-terms dominate and simplify the analysis. The fluid $\mathrm{N}_{2}$ monolayer has orientational disorder and strong positional correlations, and there is a problem of deconvoluting these processes both of which contribute to the coherent scattering. There were suggestions ${ }^{11,12}$ that the orientational disorder in a fluid phase might enable the measurement of diffusion by inelastic scattering even for a coherent scatterer. However, we find for the $\mathrm{N}_{2}$ monolayer that this occurs for such large wave vectors that the center-ofmass response function is determined by short-time ballistic motion rather than by diffusive motion.

There are long-time tails in the velocity autocorrelation functions of the $\mathrm{N}_{2}$ monolayer fluid, and they strongly influ- ence the diffusivelike motions. While the correlation functions for incoherent scattering are analyzed to give effective diffusion constants for 3D fluids, ${ }^{13}$ we find that the corresponding analysis for the monolayer fluid is much less satisfactory. Finally, we demonstrate how the single-molecule diffusive motion may be accessed despite the $\mathrm{N}_{2}$ monolayer being primarily a coherent-scattering system.

The molecule-molecule interaction is the same as that in recent work on the monolayer solids of $\mathrm{N}_{2} /$ graphite $^{14}$ and close to that for $\mathrm{N}_{2} / \mathrm{Ag}(111) .{ }^{15}$ Two models of the molecule-substrate interaction, graphite with and without corrugation, are used to explore what may be observed for a variety of substrates. The substrate is taken to be static. Ellis et al. ${ }^{9}$ in a QHAS study of low-density $\mathrm{Xe} / \mathrm{Pt}(111)$, modeled the damping of adatom motions with a Brownian friction arising from the substrate and found scarcely any effect in the linewidth for a damping coefficient of $0.25 \mathrm{ps}^{-1}$. Since the damping coefficient for $\mathrm{N}_{2} /$ graphite is estimated $^{16}$ to be in the range 2-4 $\mathrm{ns}^{-1}$, their simulations give support to the use of the static-substrate approximation in the present work.

The organization of this paper is as follows. The models and the methods of calculation are summarized in Sec. II. Results for the diffusion constants, the velocity autocorrelation function, rotational diffusion, and the incoherent scattering function are presented in Sec. III. Section IV presents an analysis of possible quasielastic scattering measurements on the finite-density fluid. Section V has some concluding remarks. Supplementary material has been deposited in the EPAPS archive. ${ }^{17}$

\section{METHODS}

The $\mathrm{N}_{2}-\mathrm{N}_{2}$ interaction, denoted X1M and defined in detail in a recent paper, ${ }^{14}$ consists of atom-atom terms, electrostatic interactions, and the McLachlan substrate-mediated interaction. The $\mathrm{N}_{2}$-substrate potential is the $\mathrm{N}_{2}$-graphite potential used there, in the corrugated-surface case, and is the lateral average of that in the smooth-surface case.

Initially, the submonolayer patches are in the form of stripes, as described in Ref. 18. A complete monolayer has 224 molecules in the cell and the densities are given as frac- 
TABLE I. Tracer diffusion constants $D_{\text {eff }}$, in $10^{-5} \mathrm{~cm}^{2} \mathrm{~s}^{-1}$, derived from mean-square displacements for the corrugated and smooth graphite models at different coverages $\rho$ using Eq. (3.1) and averaging over 150 ps.

\begin{tabular}{lccccccc}
\hline \hline \multicolumn{9}{c}{ Corrugated surface } & \multicolumn{3}{c}{$D_{\text {eff }}$} \\
$T(\mathrm{~K})$ & $\rho=3 / 14$ & $\rho=7 / 14$ & $\rho=10 / 14$ & $\rho=1.0$ & $\rho=3 / 14$ & $\rho=7 / 14$ & $\rho=10 / 14$ \\
\hline $30^{\mathrm{a}}$ & 3.3 & - & - & - & 11.4 & 4.0 & 2.8 \\
$35^{\mathrm{a}}$ & 12.8 & 3.9 & 1.1 & - & 35.5 & 8.2 & 5.4 \\
40 & 18.2 & 7.2 & 3.5 & - & 47.2 & 12.8 & 6.8 \\
45 & 21.3 & 9.4 & 4.5 & - & 50.3 & 15.0 & 7.5 \\
65 & 43.7 & 18.0 & 8.5 & - & 63.8 & 20.0 & 8.2 \\
80 & - & - & - & 4.3 & - & - & - \\
\hline \hline
\end{tabular}

${ }^{a}$ On the smooth surface, trajectory calculations show a self-bound liquid state at $30 \mathrm{~K}$. For both the corrugated and smooth surfaces, trajectory calculations indicate fluid states with large density fluctuations at $35 \mathrm{~K}$. See Ref. 19.

tions (e.g., $\rho=7 / 14$ ) of a commensurate monolayer density $\rho=1$ for graphite, $0.0636 / \AA^{2}$.

The isothermal constrained dynamics algorithm has been described previously. ${ }^{14}$ The basic time series for evaluating correlation functions is 200 ps long and averaging begins after long equilibration runs, typically ca. 800-1000 ps. Whether the monolayer is a solid, self-bound liquid, or homogeneous fluid is determined by reviewing the configurations in trajectory calculations. ${ }^{19}$ Most of the states treated in this paper are homogeneous fluids.

Although the dynamical functions may depend on the orientation of the wave vectors $\vec{q}$, the calculated dependence in these fluid phases is smaller than the fluctuations in timecorrelation functions and their Fourier transforms. Therefore, the production runs are based on 2D circular averages of $\vec{q}$ (in-plane average). This procedure reduces the numerical noise in the averaging and, for the smooth surface, reduces an artificial anisotropy introduced by the shape of the simulation cell.

\section{DYNAMICS}

\section{A. Translational diffusion}

The long-time limit of thermal motions in a monolayer fluid shows a striking persistence of velocity correlations. ${ }^{20}$ However, it has been possible to extract translational diffusion constants from simulation data, ${ }^{21}$ because there are ranges of time for which the mean-square displacement increases linearly with time and ranges of wave vector for which the single-particle dynamic structure factor is approximately a Lorentzian in frequency.

In such ranges of "long time," the mean-square displacement of the molecular centers of mass $\vec{R}_{j}$ is related to the tracer diffusion constant by ${ }^{22}$

$$
D_{\mathrm{eff}}=\lim _{t \rightarrow \infty} \frac{1}{4 t N} \sum_{j=1}^{N}\left\langle\left[\vec{R}_{j}(t)-\vec{R}_{j}(0)\right]^{2}\right\rangle .
$$

Although this definition is difficult to implement for a dense monolayer fluid in which layer promotion is occurring, ${ }^{14}$ the fitted values of $D_{\text {eff }}$ in the present work are usually ${ }^{17}$ the same to within $10 \%$ when averaging over 20 ps and over 150 ps for temperatures of $35-65 \mathrm{~K}$ and $\rho=3 / 14-10 / 14$. The values for the corrugated and smooth cases are listed in Table I. As expected, the diffusion constants in both cases decrease as coverage increases. At a given coverage, the difference between diffusion constants in the two cases narrows with increasing temperature. This is most pronounced for the $\rho=10 / 14$ density, as increase of density and of temperature both reduce the relative importance of the corrugation.

We examined plots of $\ln D_{\text {eff }}$ versus $1 / T$ for three isochores, shown in Ref. 17, for evidence of thermally activated processes in the data of Table I. There are approximately straight lines for the corrugated-surface case with slopes corresponding to an activation energy in the range 92-96 K. The potential energy barrier to $\mathrm{N}_{2}$ motion on the corrugated graphite surface is ${ }^{23} 30 \mathrm{~K}$, so the $95 \mathrm{~K}$ activation energy must include effects of the $\mathrm{N}_{2}-\mathrm{N}_{2}$ interactions in transient clusters. The $\ln D_{\text {eff }}$ vs $1 / T$ slopes for the smooth-surface case are much smaller, and the activation energy, if any, is smaller than $45 \mathrm{~K}$ for the temperature range 40-65 K.

Another determination of the diffusion constant is based on evaluation of the incoherent intermediate scattering function $F_{\text {ic }}(\vec{q}, t)$ for the center-of-mass coordinate and its Fourier transform, the incoherent dynamic structure factor $S_{\text {ic }}(\vec{q}, \omega)$ :

$$
\begin{gathered}
F_{\mathrm{ic}}(\vec{q}, t)=\frac{1}{N} \sum_{j=1}^{N}\left\langle\exp \left[\imath \vec{q} \cdot\left\{\vec{R}_{j}(t)-\vec{R}_{j}(0)\right\}\right]\right\rangle ; \\
S_{\mathrm{ic}}(\vec{q}, \omega)=\frac{1}{2 \pi} \int F_{\mathrm{ic}}(\vec{q}, t) e^{-\imath \omega t} d t
\end{gathered}
$$

To the extent that long-wavelength density fluctuations are governed by a diffusion approximation, the corresponding incoherent response function has a Lorentzian shape: ${ }^{24,25}$

$$
S_{\mathrm{ic}}(\vec{q}, \omega)=\frac{1}{\pi} \frac{D_{i} q^{2}}{\omega^{2}+\left(D_{i} q^{2}\right)^{2}}, \quad \lim q \rightarrow 0
$$




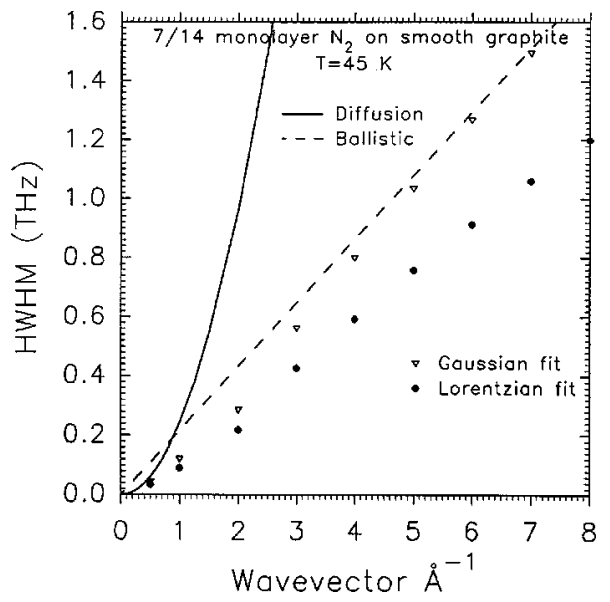

FIG. 1. The HWHM of the incoherent scattering function $S_{\text {ic }}$ for the $\rho=7 / 14$ fluid at $45 \mathrm{~K}$ for the smooth surface, as obtained from Lorentzian and Gaussian fits and from the diffusion constant $D_{\text {eff }}$ fitted to the mean-square displacement. The latter approximation only becomes accurate for $q<0.25 \AA^{-1}$.

Equation (3.4) applies to the translational motion in the fluid. The effective diffusion constant $D_{i}$ is determined by fitting the half width at half maximum (HWHM) of the incoherent scattering function and depends on the wave vector $q$.

On the other hand, for very large $q$ only the ballistic, nearly free, motion at small times is significant. Then the line shape of the scattering function is Gaussian and given by ${ }^{24}$

$$
S_{\text {ic }}(\vec{q}, \omega)=\frac{1}{\sqrt{\pi \Delta_{\text {theor }}^{2}}} \exp \left(-\frac{\omega^{2}}{\Delta_{\text {theor }}^{2}}\right),
$$

where for a classical system of masses $M$ in $2 \mathrm{D}$

$$
\Delta_{\text {theor }}^{2}=2 q^{2} \frac{k_{B} T}{M} .
$$

There is a steady evolution from the Lorentzian to the Gaussian shape for $S_{\text {ic }}$ as $q$ increases. For example, for the smooth surface with $\rho=7 / 14$ and $T=45 \mathrm{~K}$ at $q=0.5 \AA^{-1}$, the Lorentzian shape is a much better fit than the Gaussian shape, which has too little spectral strength in the wings. On the other hand, at $q=4.0 \AA^{-1}$ the converse is true and the Lorentzian shape has too much spectral strength in the wings. (These comparisons are shown in Ref. 17.) The line shape for $q>4 \AA^{-1}$ is well fitted by a Gaussian function, Eq. (3.5), and for $q>5 \AA^{-1}$ the width is within $10 \%$ of the freeparticle value given by Eq. (3.6). The transition from diffusion to the ballistic limit is shown here for the $7 / 14$ fluid at $45 \mathrm{~K}$ in Fig. 1, where the HWHM calculated with $D_{\text {eff }}$ from Table I is compared to the widths derived from fits to Lorentzian and Gaussian shapes. While an interpolation between the two line shapes has been constructed ${ }^{13}$ for $3 \mathrm{D}$ fluids, the corresponding generalized line shape did not fit the simulation data for the 2D fluid.

The Gaussian HWHM for several densities are shown in Fig. 2 for both corrugated- and smooth-surface cases. The free-particle approximation becomes accurate at smaller $q$

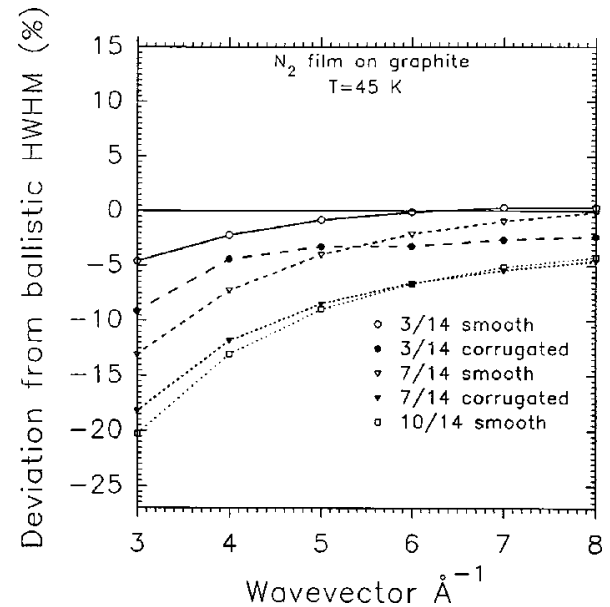

FIG. 2. The Gaussian widths (HWHM), shown as the deviation in percent from the ballistic width, determined from $S_{\text {ic }}$ for several fluid densities at $45 \mathrm{~K}$ as a function of wave vector. Open symbols are for the smooth surface and filled symbols for the corrugated surface.

for the lower densities, but the corrugation also reduces the width significantly at the smaller $q$; both of these are expected trends.

For Fig. 1, the Lorentzian shape fits $S_{\text {ic }}$ at $q=0.5 \AA^{-1}$ well, but the $D_{i}=8.4 \times 10^{-5} \mathrm{~cm}^{2} / \mathrm{s}$ is $40 \%$ less than the $D_{\text {eff }}$ derived from the mean-square displacements. Even at $q$ $=0.25 \AA^{-1}$, the $D_{i}=12 \times 10^{-5} \mathrm{~cm}^{2} / \mathrm{s}$ is $20 \%$ smaller than $D_{\text {eff }}$. Similarly, the Lorentzian fit at $q=0.5 \AA^{-1}$ for the $\rho$ $=10 / 14, \quad T=45 \mathrm{~K}$ smooth-surface case gives $D_{i}$ $=5.2 \times 10^{-5} \mathrm{~cm}^{2} / \mathrm{s}, 30 \%$ smaller than the corresponding $D_{\text {eff }}$. That is, none of these cases is truly in the $q \rightarrow 0$ limit to which $D_{\text {eff }}$ applies.

The approach to the ballistic limit for low-density Xe/ $\operatorname{Pt}(111)$ was studied in experiments and calculations by Ellis et $a l .{ }^{9}$ For $\mathrm{Xe} / \mathrm{Pt}(111)$, the ballistic approximation is already accurate at $0.5 \AA^{-1}$, perhaps because that density is only $\frac{1}{5}$ of the lowest density $\mathrm{N}_{2}$ case treated here.

\section{B. Velocity correlation functions}

The measures for the diffusion constant in Sec. III A are closely related to the autocorrelation function of the centerof-mass velocity, which for component $v_{\alpha}$ is defined by

$$
f_{\alpha}(t)=\left\langle v_{\alpha}(t) v_{\alpha}(0)\right\rangle /\left\langle v_{\alpha}(0)^{2}\right\rangle
$$

We use such $f(t)$ for another characterization of the dynamics at several $\rho$ and $T$. The case of $\rho=7 / 14$ at $T=40 \mathrm{~K}$ on the corrugated surface, shown in Ref. 17, is typical and has three stages of decay: (a) at very small times, in the first 0.1 ps, $f(t)$ decreases quadratically with time to a value of 0.9 ; (b) at intermediate times, the next $0.5 \mathrm{ps}$, there is an approximately exponential decay to a value of about 0.1 ; and (c) over the next few ps, there is a final slower decay.

The parabolic variation in stage (a) corresponds to the ballistic regime discussed for $S_{\text {ic }}$ at Eq. (3.5). The parameter 
$b$ in the variation $\exp (-b t)$ in stage (b) is the friction coefficient in a Langevin equation ${ }^{25}$ and is used to define a diffusion constant

$$
D_{\text {velexp }}=k_{B} T /(M b)
$$

that is tabulated in Ref. 17 for $\rho=3 / 14,7 / 14$, and 10/14. In each case, $D_{\text {velexp }}$ is smaller than the $D_{\text {eff }}$ derived from the mean-square displacement. This is a direct consequence of the persistence of the velocity correlations beyond the times expected from the Langevin-friction approximation and is a characteristic of 2D fluids. The $b$-value pairs for $\rho=3 / 14$ and $7 / 14$ scale with the density to about $10 \%$ in most of the temperature and corrugation cases, in accord with the binarycollision approximation to kinetic theory. ${ }^{26}$ At a given temperature $b$ increases as $\rho$ increases, in accord with the anticipated faster decay and smaller diffusion constant at higher density. There is a sharp drop in $b$ for the smooth-surface case from 30 to $35 \mathrm{~K}$ that is correlated with a change from a self-bound liquid filling only part of the cell to a homogeneous fluid filling the cell.

The very slow decay in stage (c) is referred to as "the long-time tail" of the correlation function. ${ }^{20}$ Fitting $f_{\alpha}(t)$ to a form $a / t$ gives a measure of the strength of the "tail" although there is much scatter in plots of $t f(t)$. The $a$ value decreases as the density increases and is insensitive to temperature except for the lowest temperatures where there are self-bound liquid patches for the smooth surface. Corrugation of the holding potential causes a great reduction of $a$, but the relative effect in the time constants $b$ is much smaller.

\section{Rotational diffusion}

Much of the monolayer phase diagram of physisorbed $\mathrm{N}_{2}$ consists of plastic solid and fluid states that are orientationally disordered. The simulations enable quantitative characterizations of the dynamics of the orientational degree of freedom of the molecule.

The incoherent-atomic scattering function $F_{\text {ia }}$ for a rigid diatomic molecule $\left(N_{a}=2\right)$ is $^{27}$

$$
F_{\mathrm{ia}}(\vec{q}, t)=\sum_{j=1}^{2}\left\langle\exp \left[\imath \vec{q} \cdot\left\{\vec{r}_{j}(t)-\vec{r}_{j}(0)\right\}\right]\right\rangle / N_{a}
$$

where the atomic positions $\vec{r}_{j}$ are expressed in terms of the center-of-mass position $\vec{R}$, the internuclear distance $l$ and the orientation of the internuclear axis $\hat{n}$ by

$$
\vec{r}_{j}=\vec{R} \pm(l / 2) \hat{n} .
$$

Define a correlation function $F_{i<}$ that isolates the orientational coordinate

$$
F_{i<}(\vec{q}, t)=\langle\exp [\imath l \vec{q} \cdot\{\hat{n}(t)-\hat{n}(0)\} / 2]\rangle .
$$

In many cases the center-of-mass and orientational motions are nearly decoupled, ${ }^{11,12}$

$$
F_{\mathrm{ia}}(\vec{q}, t) \simeq F_{\mathrm{ic}}(\vec{q}, t) F_{i<}(\vec{q}, t),
$$

TABLE II. Comparison of rotational diffusion time constants on the corrugated surface. The time constants $(k=1)$ of exponential relaxation of the orientational self-correlation function in ps evaluated at $1 \AA^{-1}$ are listed.

\begin{tabular}{lcc}
\hline \hline$T(\mathrm{~K})$ & $\rho=7 / 14$ & $\rho=1.0$ \\
\hline 25 & - & 9.8 \\
30 & - & 3.8 \\
45 & $0.9^{\mathrm{a}}$ & - \\
50 & - & 0.9 \\
\hline \hline
\end{tabular}

${ }^{\mathrm{a}}$ On the smooth surface at $\rho=7 / 14$, the time constant is $1.0 \mathrm{ps}$.

with $F_{\text {ic }}$ defined in Eq. (3.2). Equation (3.12) is satisfied to a good approximation in the simulations of the plastic phase of the monolayer solid and in the monolayer fluid, as tested for $q=0.5-5 \AA^{-1}$ and times up to 5 ps.

When $\hat{n}$ remains in the monolayer plane, $F_{i<}$ has the form $^{24}$

$$
\begin{gathered}
F_{i \angle}(t)=\left[J_{0}(Q)\right]^{2}+2 \sum_{k=1}^{\infty}\left[J_{k}(Q)\right]^{2} C_{k}(t), \\
C_{k}(t)=\langle\cos \{k[\phi(t)-\phi(0)]\}\rangle .
\end{gathered}
$$

The circular average in Eq. (3.11) corresponds to random azimuthal angles $\phi(0)$ and was performed in the simulation to enhance the stability of the averaging process. The $J_{k}$ are cylindrical Bessel functions of argument $Q=q l / 2$.

The monolayer fluid simulations for $\mathrm{N}_{2}$ show Eq. (3.13) is quite accurate. One test is to compare the long-time limit ' $t \rightarrow \infty$,' where the time-dependent terms in Eq. (3.13) have decayed to zero, to $\left[J_{0}(Q)\right]^{2}$. For the $\rho=7 / 14$ fluid on the corrugated surface at $45 \mathrm{~K}$, the simulation agrees with this to within $10 \%$ up to $q \approx 2.5 \AA^{-1}$ and the deviations are attributed to the increasing significance of out-of-plane fluctuations of the molecular axis as $q$ increases. A second test is that, for ranges of $q$ for which the $J_{1}$ term dominates the sum, the decay time of the averaged $F_{i<}$ is independent of $q$. This is confirmed by forming $\Delta F(q, t)=F_{i<}(t)-F_{i<}(\infty)$ and showing that the ratio $\Delta F(q, t) / \Delta F(q, 0)$ is nearly independent of $q$ for times up to 3 ps and wave vectors up to $2 \AA^{-1}$. A third test is based on scaling the Fourier transform of the time-dependent term in Eq. (3.13) for wave vectors $q=0.5-2.5 \AA^{-1}$. The frequency shapes of the curves should be (and are) identical when the $k=1$ term dominates, differing only by the scale factor $\left[J_{1}(Q)\right]^{2}$. This scaling is illustrated for the monolayer solid $(\rho=1)$ on graphite at $25 \mathrm{~K}$ and $30 \mathrm{~K}$ in Ref. 17.

The fitted time constants of the exponential decay $\exp \left(-t / t_{\llcorner}\right)$are listed in Table II for the $\rho=7 / 14$ monolayer fluid at $45 \mathrm{~K}$ and for the commensurate monolayer solid $\rho$ $=1$ at several temperatures. The orientational-disordering temperature calculated ${ }^{14}$ with the present model is $22 \mathrm{~K}$ so that the $25 \mathrm{~K}$ entry presumably reflects a solid with longlived orientational fluctuations. The simulation captures the sequential nature of the disordering of the monolayer $\mathrm{N}_{2}$, with rotational diffusion from orientational disorder in the plastic crystal beginning before the onset of translational dif- 
fusion in the fluid. The $t_{\angle}$ data in Table II for the monolayer solid at temperatures up to $10 \mathrm{~K}$ above its orientational disorder transition have a strong $T$ dependence that might give valuable information on this subtle transition. ${ }^{1}$

The values in Table II for the monolayer fluid are in the range found for other dense phases of disordered molecules. The $k=1$ orientational relaxation time for $3 \mathrm{D}$ liquid $\mathrm{N}_{2}$ at 66 $\mathrm{K}$ is ${ }^{12} 0.7 \mathrm{ps}$ and for a monolayer solid of $\mathrm{CH}_{4}$ /graphite at $55 \mathrm{~K}$ is ${ }^{10} 0.8 \mathrm{ps}$.

The function $C_{k}(t)$ has been evaluated in previous $\mathrm{N}_{2}$ simulations at $\rho=1$ and temperatures overlapping the range treated here, although there are small differences in the interaction models. The qualitative difference in behavior below and above the orientational disordering temperature was demonstrated ${ }^{28}$ with simulations at $16 \mathrm{~K}$ and $40 \mathrm{~K}$. At $40 \mathrm{~K}$, fitting an exponential to the $k=1$ simulation data gives $t_{\angle}$ $\simeq 1.5$ ps. At $74 \mathrm{~K}$, for what appears to be a monolayer fluid, fitting the exponential to the $k=1$ simulation data ${ }^{29}$ gives $t_{L} \simeq 0.35 \mathrm{ps}$. However, the decay time does not scale as $k^{2}$ when the $k=2$ data $^{29}$ are treated and the main features of $C_{k}(t)$ at $74 \mathrm{~K}$ appear to be represented better by free rotation, in analogy to the ballistic approximation for translation, Eq. (3.5).

\section{QUASIELASTIC SCATTERING}

The functions treated in Sec. III are those where it is simplest to identify and quantify diffusion in the calculations. However, most of the experimental methods use scattering to measure the intermediate scattering function or the dynamic structure factor. In this section we summarize calculations done to identify wave vector ranges where the center of mass and rotational correlation functions may be probed for a coherent-scattering target such as $\mathrm{N}_{2}$.

The coherent-atomic intermediate scattering function $F_{\text {ca }}$ for the diatomic molecular system is defined by

$$
\begin{aligned}
F_{\text {ca }}(\vec{q}, t)= & \frac{1}{\left(N_{a} N_{\mathrm{mol}}\right)} \sum_{j, \alpha} \sum_{j^{\prime}, \alpha^{\prime}}\left\langle\exp \left\{\imath \vec{q} \cdot\left[\vec{R}_{j}+\vec{\rho}_{j, \alpha}\right](t)\right\}\right. \\
& \left.\times \exp \left\{-\imath \vec{q} \cdot\left[\vec{R}_{j^{\prime}}+\vec{\rho}_{j^{\prime}, \alpha^{\prime}}\right](0)\right\}\right\rangle,
\end{aligned}
$$

with $j, j^{\prime}$ sums over the number of molecules $N_{\text {mol }}=N$ and $\alpha, \alpha^{\prime}$ sums over the two atoms, $N_{a}=2$, of a given molecule. The positions relative to the center-of-mass $\vec{R}_{j}$ are $\vec{\rho}_{j, \alpha}=$ $\pm(l / 2) \hat{n}_{j}$. The corresponding function for the center-ofmass degree of freedom is

$$
F_{\mathrm{cc}}(\vec{q}, t)=\frac{1}{N_{\mathrm{mol}}} \sum_{j} \sum_{j^{\prime}}\left\langle\exp \left\{\imath \vec{q} \cdot \vec{R}_{j}(t)\right\} \exp \left\{-\imath \vec{q} \cdot \vec{R}_{j^{\prime}}(0)\right\}\right\rangle .
$$

The Fourier transforms of $F_{\mathrm{ca}}$ and $F_{\mathrm{cc}}$ are denoted $S_{\mathrm{ca}}(\vec{q}, \omega)$ and $S_{\text {cc }}(\vec{q}, \omega)$, respectively.

The dynamic structure factor $S_{\mathrm{ca}}(\vec{q}, \omega)$ gives the coherent response of the correlated system in inelastic scattering experiments. For neutron scattering, the total dynamic structure factor has terms from the coherent $\left(\sigma_{c}\right)$ and incoherent $\left(\sigma_{i}\right)$ neutron cross sections and is given in terms of the Fourier transforms of Eqs. (4.1) and (3.9) by

$$
S(\vec{q}, \omega)=\sigma_{c} S_{\mathrm{ca}}(\vec{q}, \omega)+\sigma_{i} S_{\mathrm{ia}}(\vec{q}, \omega) .
$$

Since $^{30} \sigma_{i} / \sigma_{c}=0.045$ for low-energy neutron scattering from ${ }^{14} \mathrm{~N}, S(\vec{q}, \omega)$ is dominated by the coherent-scattering term for most of the $q$ range. In some circumstances, ${ }^{31}$ the relative contribution of the $\sigma_{i}$ term is enhanced by experiments at small $q$. This does not seem to be a promising method of probing incoherent processes in the $\mathrm{N}_{2}$ liquid, because the liquid structure factor of the submonolayer fluid remains quite appreciable at small $q$ for the temperatures treated here.

However, there are special wave vectors ${ }^{11,12}$ where $F_{\text {ca }}$ is dominated by the incoherent response described with $F_{\text {ia }}$, because the form factor of the diatomic molecule vanishes and thus eliminates the contribution from the coherent center-of-mass scattering. To explore what may be learned with quasielastic scattering from the $\mathrm{N}_{2}$ monolayer fluid, we have compared the simulation results for Eq. (4.1) to an approximate form using the incoherent response functions.

If the center-of-mass and orientational degrees of freedom are statistically independent, as assumed in Eq. (3.12), and, further, if the orientations of different molecules are not correlated, Eq. (4.1) reduces to

$F_{\mathrm{ca}}(\vec{q}, t) \simeq 2\langle\cos [\vec{q} \cdot(l / 2) \hat{n}]\rangle^{2} F_{\mathrm{cc}}(\vec{q}, t)+F_{\mathrm{ic}}(\vec{q}, t) \Delta F_{i<}(\vec{q}, t)$,

where, using circular averaging and $Q=q l / 2$,

$$
\Delta F_{i<}(\vec{q}, t)=4 \sum_{k=1}^{\infty}\left[J_{2 k}(Q)\right]^{2} C_{2 k}(t)
$$

and

$$
\langle\cos [\vec{q} \cdot(l / 2) \hat{n}]\rangle^{2}=\left[J_{0}(Q)\right]^{2} .
$$

We made extensive tests of the approximation for $S_{\mathrm{ca}}(\vec{q}, \omega)$ obtained by Fourier transformation of Eq. (4.4) for many density and corrugation combinations. The $q=5 \AA^{-1}$ case of the $\rho=7 / 14, T=45 \mathrm{~K}$ smooth-surface fluid is shown in Fig. 3. There, as expected from the near vanishing of $J_{0}(q l / 2)$, the $S_{\text {ca }}$ is well fitted by the term arising from the second term on the right-hand side of Eq. (4.4). The HWHM of $1.25 \mathrm{THz}$ agrees with that obtained by convoluting the ballistic approximation to $F_{\text {ic }}$ with an exponential ( $\tau$ $=0.53 \mathrm{ps}$ ) fit to $\Delta F_{i \angle}$ over the first $1.0 \mathrm{ps}$. (There is a slower nonexponential decay of $\Delta F_{i<}$ from 2 to 4 ps.) In experiments, a fit to $S_{\mathrm{ca}}(\vec{q}, \omega)$ at such $q$ might be used to determine the rotational relaxation time $\tau$. There are marked changes in fitting the frequency dependence of $S_{\mathrm{ca}}(\vec{q}, \omega)$ for $q=4$ and $6 \AA^{-1}$, which bracket this $q$, as shown in Ref. 17 . In the small- $q$ range, the calculated $S_{\text {ca }}$ is well fitted up to $q \approx 2 \AA^{-1}$ by the first term on the right-hand side, and the second term is very small. ${ }^{32}$ Indeed, in calculations for the commensurate monolayer solid, nearly identical values of 


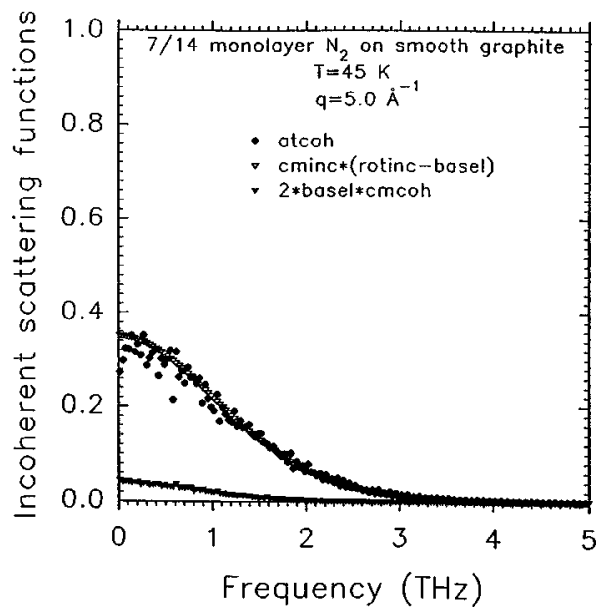

FIG. 3. Comparison of simulation data for the coherent-atomic dynamic structure factor $S_{\mathrm{ca}}(\vec{q}, \omega)$ to the transform of the product of the incoherent center-of-mass function $F_{\text {ic }}$ and $\Delta F(i, \angle)$. Results are shown for the $\rho=7 / 14$ smooth-surface fluid at $45 \mathrm{~K}$ at $q$ $=5 \AA^{-1}$. The planar rotor model predicts that the contribution of $S_{\mathrm{cc}}(\vec{q}, \omega)$ should vanish at $q=4.38 \AA^{-1}$, Sec. IV. The filled dots denote the values of $S_{\mathrm{ca}}$; the open triangles denote the results of the Fourier transform of the product $F_{\text {ic }} \Delta F(i, \angle)$; the full triangles denote $2\langle\cos [\vec{q} \cdot(l / 2) \hat{n}]\rangle^{2} S_{\mathrm{cc}}$, the product of the center-of-mass coherent term and the $t \rightarrow \infty$ baseline of the rotational function. Much poorer correspondence between $S_{\mathrm{ca}}(\vec{q}, \omega)$ and the transform of $F_{\text {ic }} \Delta F(i, \angle)$ is obtained at $q=4$ and $6 \AA^{-1}$.

the Brillouin-zone-center gap were derived from the atomic and the center-of-mass correlation functions. ${ }^{14}$

The explicit forms in Eqs. (4.5) and (4.6) depend on the molecular axes remaining in the monolayer plane. As discussed for Eq. (3.13), this is a good approximation, although the corrugated surface and molecule-molecule interactions both lead to some tipping of axes out of the plane. For $\rho$ $=3 / 14$ and the smooth surface, the response at $q \approx 4.5 \AA^{-1}$ is dominated by the incoherent terms, while for the corrugated surface the corresponding $q$ is a bit larger. The simulation shows that these large $q$ values do not affect the width arising from the rotational relaxation $\left(\Delta F_{i \angle}\right)$ but the $F_{\text {ic }}$ factor then is dominated by the ballistic translational motion.

At small wave vectors, where $S_{\mathrm{ca}}(\vec{q}, \omega)$ follows $S_{\mathrm{cc}}(\vec{q}, \omega)$ closely, it seems feasible to obtain a fair approximation to the $q$-dependent diffusion constant $D_{i}$. The Vineyard approximation $^{33}$ for the time-dependent pair distribution function leads to an approximation

$$
F_{\mathrm{cc}}(\vec{q}, t) \propto S(\vec{q}) F_{\mathrm{ic}}(\vec{q}, t)
$$

where $S(\vec{q})$ is the static structure factor of the $2 \mathrm{D}$ fluid. This approximation was tested for the $\rho=7 / 14, T=45 \mathrm{~K}$ fluid on the smooth surface by fitting the time dependence of $F_{\text {ia }}$ and of $F_{\text {cc }}$ on $0.5-5.0$ ps to exponentials for $q=0.5$ and $1.0 \AA^{-1}$.
The time constants are $\approx 10 \%$ smaller than those obtained from the Lorentzian fits to $S_{\text {ic }}$ discussed in Sec. III A, and the deviation is larger at the larger $q$. This is quite encouraging for using the shape of the coherent response function $S_{\mathrm{ca}}(\vec{q}, \omega)$ at small $q$ to measure diffusion in the monolayer fluid. Future calculations might test how the accuracy of the Vineyard approximation changes with density.

\section{CONCLUSION}

The mean-square displacements, velocity autocorrelation functions, and incoherent scattering functions were calculated for the monolayer $\mathrm{N}_{2}$ fluid. The diffusion constants on the corrugated surface are smaller than those on the smooth surface and reflect a thermally activated motion (energy $\approx 95 \mathrm{~K}$ ) not evident in the latter. Much of the strength of the velocity autocorrelation functions is in time ranges not well fitted by the exponential time dependence expected for Langevin kinetics. The several measures of the center-ofmass translation are all strongly influenced by the persistence of correlations ("long-time tails") of 2D fluids, even though the $\mathrm{N}_{2}$ molecules in these simulations may tip out of the adsorption plane and may desorb to a second-layer gas phase or to 3D gas.

At intermediate- and short-time scales, motions in the monolayer fluid are qualitatively similar to those in 3D fluids, with the Lorentzian and Gaussian shapes for spectral functions expected for diffusive and ballistic motions, respectively. However, for a wide range of wave vectors, the widths of the spectral functions are not simply given by the mean-square displacements either for diffusive motion or free motion. In the examples treated here, the Lorentzian line shape is reached for $q=0.5 \AA^{-1}$, but the width is $30-40 \%$ smaller than that estimated using the mean-square displacements. The ballistic approximation to the width becomes accurate to only $5-10 \%$ for $q=5 \AA^{-1}$ and becomes accurate at smaller $q$ for lower densities. For the present corrugations and temperatures, the widths of the spectral functions are not very sensitive to the corrugation. The widths derived from quasielastic experiments on monolayer fluids probably will not be easily interpreted in terms of the limiting approximations, and there will need to be concurrent simulations at the experimental conditions to make full use of the experimental data.

\section{ACKNOWLEDGMENTS}

We thank Professor H. Taub for many helpful discussions of this work. The work was partially supported by the National Science Foundation under Grant No. DMR-9807410 (L.W.B.) and by The Danish Natural Science Foundation (F.Y.H.). L.W.B. thanks the Department of Chemistry and the Technical University of Denmark for hospitality during much of the time this work was done. 
${ }^{1}$ D. Marx and H. Wiechert, Adv. Chem. Phys. 95, 213 (1997).

${ }^{2}$ G.S. Leatherman and R.D. Diehl, Langmuir 13, 7063 (1997).

${ }^{3}$ C. Ramseyer, C. Girardet, F. Bartolucci, G. Schmitz, R. Franchy, D. Teillet-Billy, and J.P. Gauyacq, Phys. Rev. B 58, 4111 (1998).

${ }^{4}$ A. Marmier, C. Ramseyer, P.N.M. Hoang, C. Girardet, J. Goerge, P. Zeppenfeld, M. Büchel, R. David, and G. Comsa, Surf. Sci. 383, 321 (1997).

${ }^{5}$ P. Zeppenfeld, R. David, C. Ramseyer, P.N.M. Hoang, and C. Girardet, Surf. Sci. 444, 163 (2000).

${ }^{6}$ K.W. Herwig, Z. Wu, P. Dai, H. Taub, and F.Y. Hansen, J. Chem. Phys. 107, 5186 (1997).

${ }^{7}$ M. Bienfait, B. Asmussen, M. Johnson, and P. Zeppenfeld, Surf. Sci. 460, 243 (2000).

${ }^{8}$ F.Y. Hansen, K.W. Herwig, B. Matthies, and H. Taub, Phys. Rev. Lett. 83, 2362 (1999); D. Fuhrmann, L. Criswell, H. Mo, U.G. Volkmann, K.W. Herwig, H. Taub, and F.Y. Hansen, Physica B 276-8, 345 (2000).

${ }^{9}$ J. Ellis, A.P. Graham, and J.P. Toennies, Phys. Rev. Lett. 82, 5072 (1999).

${ }^{10}$ P. Thorel, J.P. Coulomb, and M. Bienfait, Surf. Sci. 114, L43 (1982)

${ }^{11}$ V.F. Sears, Can. J. Phys. 44, 1279, 1299 (1966).

${ }^{12}$ K. Carneiro and J.P. McTague, Phys. Rev. A 11, 1744 (1975).

${ }^{13}$ R.K. Hawkins and P.A. Egelstaff, Mol. Phys. 29, 1639 (1975); P.A. Egelstaff and P. Schofield, Nucl. Sci. Eng. 12, 260 (1962); J.P. Boon and S. Yip, Molecular Hydrodynamics (McGraw-Hill, New York, 1980), Sec. 4.5.

${ }^{14}$ F.Y. Hansen and L.W. Bruch, Phys. Rev. B 51, 2515 (1995).

${ }^{15}$ L.W. Bruch and F.Y. Hansen, Phys. Rev. B 57, 9285 (1998).

${ }^{16}$ R.D. Boutchko and L.W. Bruch, Phys. Rev. B 59, 10992 (1999).

${ }^{17}$ See EPAPS Document No. E-PAPS: E-PRBMDO-64-030128 for supplementary material, five composite figures, one table, and some discussion. E-PAPS document files may be retrieved free of charge from our FTP server (http://www.aip.org/pubservs/ paps.html) or from ftp.aip.org in the directory /epaps/. For further information, e-mail: paps@aip.org or fax: 516-576-2223.
${ }^{18}$ F.Y. Hansen, L.W. Bruch, and H. Taub, Phys. Rev. B 52, 8515 (1995).

${ }^{19}$ F.Y. Hansen (unpublished).

${ }^{20}$ B.J. Alder and T.E. Wainwright, Phys. Rev. A 1, 18 (1970); T.E. Wainwright, B.J. Alder, and D. Gass, ibid. 4, 233 (1971).

${ }^{21}$ S. Ranganathan, G.S. Dubey, and K.N. Patahak, Phys. Rev. A 45, 5793 (1992).

${ }^{22}$ The notation $D_{\text {eff }}$ is used by E. Leutheusser, D.P. Chou, and S. Yip, J. Stat. Phys. 32, 523 (1983).

${ }^{23}$ F.Y. Hansen, L.W. Bruch, and S.E. Roosevelt, Phys. Rev. B 45, 11238 (1992).

${ }^{24}$ T. Springer, Quasielastic Neutron Scattering for the Investigation of Diffusive Motions in Solids and Liquids, Vol. 64 of Springer Tracts in Modern Physics (Springer-Verlag, Berlin, 1972).

${ }^{25}$ W. Marshall and S.W. Lovesey, Theory of Thermal Neutron Scattering (Oxford University Press, Oxford, 1971).

${ }^{26}$ J.O. Hirschfelder, C.F. Curtis, and R.B. Bird, Molecular Theory of Gases and Liquids (Wiley, New York, 1954).

${ }^{27}$ For free $2 \mathrm{D}$ translation and rotation of the diatomic molecule, the Fourier transform of $F_{\text {ia }}$ is a Gaussian, analogous to Eq. (3.5), with a width $\Delta^{2}=2 k_{B} T q^{2} /\left(\frac{4}{3} m_{\mathrm{N}}\right)$, where $m_{\mathrm{N}}$ is the atomic mass. The free 3D rotor, treated by P.G. de Gennes, Physica (Utrecht) 25, 825 (1959), has $\frac{6}{5}$ in place of $\frac{4}{3}$.

${ }^{28}$ J. Talbot, D.J. Tildesley, and W.A. Steele, Mol. Phys. 51, 1331 (1984); R.M. Lynden-Bell, J. Talbot, D.J. Tildesley, and W.A. Steele, ibid. 54, 183 (1985).

${ }^{29}$ A.V. Vernov and W.A. Steele, Langmuir 2, 606 (1986).

${ }^{30}$ J. Byrne, Neutrons, Nuclei, and Matter (Institute of Physics, Bristol, 1995)

${ }^{31}$ S.J. Cocking, J. Phys. C 2, 2047 (1969).

${ }^{32}$ J.J. Weis and D. Levesque, Phys. Rev. A 13, 450 (1976), in calculations for 3D liquid $\mathrm{N}_{2}$ at $70 \mathrm{~K}$, also found that $F_{\text {ca }}$ has the same shape as $F_{\text {cc }}$ up to $q \approx 2 \AA^{-1}$.

${ }^{33}$ G.H. Vineyard, Phys. Rev. 110, 999 (1958); A.P. Graham, W. Silvestri, and J.P. Toennies, in Surface Diffusion: Atomistic and Collective Processes, edited by M.C. Tringides (Plenum, New York, 1997), p. 565. 